\title{
Paradigm Shifts in Human-Canine Synergy and Training
}

\author{
Theo Koutsopoulos ${ }^{1}$ and K C Koutsopoulos ${ }^{2 *}$ \\ ${ }^{1}$ American Community School, Athens, Greece \\ ${ }^{2}$ National Technical University of Athens, Greece
}

Submission: September 26, 2019; Published: November 04, 2019

*Corresponding author: K C Koutsopoulos, National Technical University, Athens, Greece

\begin{abstract}
In order to deal with Human-Canine Synergy (HCS), which includes canine training, there is a fundamental need to accept a scientific approach in terms of both the way HCS and training are regarded and are practiced. To substantiate that need, this paper provides a five-prong proposition supporting the position that HCS and canine training have recently undergone paradigm shifts from the present behavioral-based paradigm, which in turn has replaced the simple traditional paradigm, to the new scientific paradigm, bringing HCS and canine training into a new approach which is based on utilizing scientific concepts and principles in explaining and describing the world humans and canines share. To illustrate the new paradigm an example is presented explaining the way canines can be trained to restrain their aggression by utilizing scientific knowledge and which results in an extremely effective, efficient and mainly meaningful canine training approach.
\end{abstract}

\section{Introduction}

In the last few years, important differentiations have occurred related to the way we view social issues as well as their basic principles. There are three important differentiations related to canine training as part of the human-canine synergy (HCS), which is a concept recently introduced to indicate the mutually beneficial and dynamic affiliation of humans and canines [1].

i. From the assumption that relationships, including HCS, exist in order to be discovered, to the perception that they constitute social constructs that are our own creations.

ii. From considering any social activity as an externally given creation, to the faith that a system exists that includes HCS and canine training, within which all its participating actors are related and interact in an integrating process that incorporates physical, social, cultural etc. factors.

iii. From the perception that there is a unique, exclusively behavioral, way to approach men's' "best friends", to the realization that any approach towards them, including their training, has to follow the laws and rules that determine the world they both shares.

These changes are of importance to HCS, because they clearly show the need for a different approach of human activities to wards canine use and training, by putting emphasis on the principles upon which the human environment is based and operate. The literature [1-3] and experience show that the way humans deal with and practice their activities towards canines are determined almost exclusively by the "myths" associated with them. These myths work as instruments not only in clarifying the fields of their perception but allowing them to be able to deal with only certain problems and not seeing the others [4]. In this way their myths give humans the necessary intellectual calm for their judgments to be revealed in their minds as exact reflections of reality, which by necessity must follow objective/scientific rules.

If this is the case, then a major concern in dealing with canine training, as part of HCS, is the source of the training myths. Most certified dog trainers accept the notion that all canine related approaches should satisfy certain functional conditions, requiring systematic approaches in order to satisfy basic methodological needs. These conditions and needs in turn are the result of the values that the canine concerned community accepts and they constitute the basis for the way each member and the community as a whole face canine training. It is essentially this system of values that justifies any training approach and provides the instrumental force that formulates the theoretical and practical base of canine training. From this perspective, it should be clear that in order to 
establish the right approach in considering canine training, it is necessary the reality of canine training myths to be confronted. Because in this way a creative contribution can be achieved towards the appropriate training objectives, which in turn constitute an inseparable part of the canine community's envisagement of canine training as well as of the methodological approaches utilized.

As a result, the questions that need to be asked in the current social, economic, cultural etc., conditions are: which are the "myths" and the "values" with which canine training must be approached? Categorically and without any unambiguity, this paper declares that at the center of the approach towards canine training should be the application of scientific concepts, as this constitutes the source of the canine community myths and values not only in terms of the way HCS and canine training should be regarded, but also with respect to the training methodologies utilized. This leads to the position that present approaches to canine training, are now absolute and canine training is in the period, of what is termed in this paper, the scientific paradigm.

More specifically, the position presented here is simple in explaining and justified it, but radical when considering it in terms of the current practices of canine training. That is, certified canine trainers have gained the acceptance of the canine community (dog owners, breeders, academics etc.) that they have the knowledge and background to evaluate the character and temperament of canines and analyze their behavior as well as present the results of their analyses for an efficient and effective canine training. But these acts and processes cannot be dealt with unless we accept the fact that they represent different manifestations of "a whole", the dialectic entity of HCS. Therefore, to approach canine training is possible only through an examination of the nature and the evolution of the human-canine synergy, which in turn determines how men's "best friends" are perceived as well as how they are trained. This objective represents the focus of this paper, which is a continuation of the work presented in [1], that examines HCS and canine training paradigm shifts not exclusively in terms of the methods used, as has been done up to now [5], but on how we perceive and operationalize the developing societal and HCS conditions. More specifically, the paper's contribution is presented in the form of the following proposition.

\section{The Proposition}

Nowadays for an appropriate way to deal with canine training as part of the human-canine synergy there is an axiomatic need to accept a new approach both in terms of the way canines are regarded and of how their training can be accomplished. The new approach represents a five-prong proposition: first, the determining factor in the observed changes in HCS and canine training is the result of the changes in societal perceptions and beliefs; second, that canine training is in the process of a new paradigm shift; third, the paradigm shifts have been catalytic in altering the way behavioral modification is approached in canine training; fourth, that human-canine synergy, which includes dog training, is by necessity holistic; and fifth that the new paradigm should be understood as representing the confrontation of HCS and canine training with the scientific principles of the real world. The proposition, as outlined, clearly indicate that in order to understand the role, the value and the impact of canine training and mainly their underpinned behavioral base, it is imperative that all these aspects are examined.

The first aspect suggests that the driving forces in the observed changes towards HCS and canine training are the result of the shifts in societal perceptions and beliefs. Indeed, it is universally accepted and well documented that societal values and goals are changing through time resulting in profound changes in all aspects of our lives [6], including the way HCS and canine training are regarded and applied. In very general terms the following HCS and canine training changes have been observed (Figure 1 left column):

i. $\quad$ For many years and up to the end of World War II HCS was practically of no concern to the society. Canines had a limited individual and societal value and canine training was a hobby for a few true canine lovers. Canines were simply considered as a low-level instrument in attaining other pressing personal and in a few cases societal objectives.

ii. It was practically only after the end of WW II that the significance of HCS and canine training was recognized, and they acquired a place in the societal interests. The affiliation between humans and canines reached societal recognition by focusing on canines assisting or working for humans. At the same time canine training became necessary for canines to perform various tasks, help humans in various activities, or in general operating as tool in performing various useful to humans' activities. To avert possible objections, it should be noted that the synergy of humans and canines, in the form of dogs aiding men for their subsistence and survival as well as in everyday chores, started in the hunter-gatherer era and has been operating continuously. But it is in the second half of the 20th century that society as a whole and in various forms has explicitly recognized the role and value of canines.

iii. Finally, in the last few decades society realized that the world that surrounds us is simultaneously ecological, economic, social, technical/technological, political, cultural etc., in dialectic harmony with all aspects of the environment (natural and manmade), an integral part of which are the canines and consequently their training. As a result, the new societal concerns have moved societal responses in dealing with HCS problems and challenges, including canine training, towards an approach based on the concept that the environment humans live on and perform various activities represents a dialectic entity that consists of the many elements mentioned previously and an expression of which is canine training (Figure 1). 


\section{Psychology and Behavioral Science International Journal}

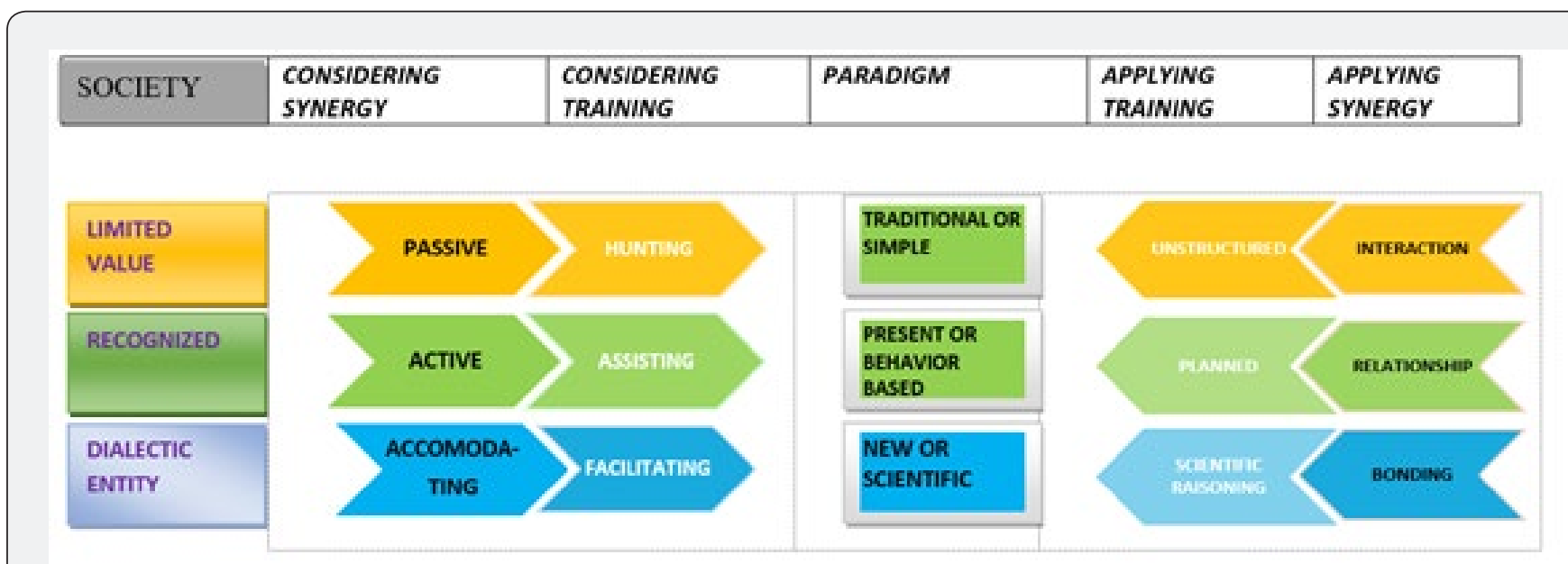

Figure 1: Considering and Practicing Human-canine Synergy and Canine Training.

The second aspect implies that two stepwise changes in canine training have taken place, which were determined by the way HCS was considered and applied over time and which in turn were the result of the societal changes that have occurred. In other words, societal changes through time have been the determining factor in shaping the responses in considering and applying HCS and canine training. These changes represent what epistemologist Kuhn [7] has termed paradigm shifts. It should be noted that changing paradigms are not rare or unexpected events in considering animals and especially canines $[8,9]$.

The third aspect is concerned with the changes in the way training goals are achieved within the HCS framework and are since canine training by necessity is related to behavioral modification. More specifically, behavioral modification has changed from a simple one-dimensional approach focusing on the most elementary aspects of canine behavior and related to obeying commands, to a more sophisticated multi-dimensional approach, where composite and more intricate canine behaviors are the object of training and lead into changing them in order to perform useful tasks, to finally altering canine behavior utilizing scientific training approaches, where the changing of canine behavior should facilitate canine contribution to human activities.

The fourth aspect of the proposition is relatively easy to comprehend, but in direct contrast with the prevailing practices. That is, within today's HCS framework, canine training should change to provide combined, simultaneous and not fragmented techniques and methods. But such an approach cannot be applied unless a major fact is accepted, namely that training should confront the various manifestations of the dialectic entity of HCS, to which training is a major part. Therefore, a holistic approach towards canine training is imperative to address present-day social, economic, cultural etc. conditions.

The fifth aspect suggests that the new scientific paradigm as a connection between HCS and science, accommodating human needs and a bonding type of synergy, should be understood as representing the confrontation of HCS and training with the real world. But out of this gloss confrontation rises a situation of the world as it exists today, which is too broad to be handled by mono or multi-dimensional approaches as well as by unstructured or planned training methods, with no regard for the holistic nature of that world. That is, the purpose of the scientific paradigm is more than just to address questions that transect behavioral analyses or integrate methods in achieving canine training. It involves an articulate spectrum of principles to help HCS and canine training to determine when and how to confront the world by seeking out a holistic approach to inter-relations and inter-dependencies, that can be achieved with the use of scientific reasoning. Therefore, it should be evident that in order to substantiate the move towards a new paradigm, there is a need to examine the current consideration of canines and the approaches of training them as well as the way they have reached through time their present form.

\section{Considering HCS and Canine Training}

The way canine training is viewed, by necessity follow changes in the way HCS is considered, which in turn is determined by the way societal values are constructed and are considered as societal goals. Every time a political, environmental, technological and in general a societal change is happening, HCS adjusts to these new conditions. However, every time a change in the HCS is happening a new approach is required to train canines. This implies that over time a stepwise process of training changes has been formulated to respond to societal changes and HCS adjustments. In considering HCS and canine training through time, the following three approaches were in operation (Figure 1, considering synergy and training columns), which represent the outstanding, typical or principal form of dog training at each period without ignoring observed exceptions. 


\section{The Traditional Approach}

In the first and long-lasting period, the limited societal concern for canines has led into considering the human-canine interaction as an insignificant process, within which the role of canines was extremely passive. In addition, because society and individuals had little use and interest in canines, HCS was restricted to the circles of the gentry who were the only ones concerned with the role and well been of canines, as well as who could afford the time and cost of their training. At the same time, the minimal societal attention to canines and their passive role in the HCS provided a mono-dimensional approach to training, which was focused mainly on hunting dogs such as pointers and setters $[10,11]$. Of course, around 127-116 B.C. farmer Marcus Varro, was recorded as advising his fellow Romans on raising and training puppies for herding livestock [12], while in 1792 an asylum in England introduced caring for animals as a therapeutic approach to assist patients with psychological, anxiety and stress problems [13]. But at this long time period, the only organized and accepted canine training has been related to hunting, providing the impetus of the traditional training paradigm.

\section{The Existing Approach}

After WW II, the beginning of the second period, the significance and utility of canines in the lives of common people started to be recognized, resulting in canines acquiring a place in the societal interests. In addition, there was strong questioning of the mono-dimensional and fragmented approaches within HCS, resulting in the development of alternative ways in considering the inter-relationship between humans and canines. More specifically, all human needs for canines were required to be approached from various perspectives and concerns $[1,14]$. HCS was treated as if it consisted of distinct parts of a multi-facet reality that led to a multi-dimensional approach towards HCS. As a result, during this time period HCS not only reached prominence, but it was considered as a composite process, requiring canines to have an active role focusing on real-life issues related to canines assisting or working for humans. Basically, canines were performing various tasks or help humans in various activities.

During that time period, the increase of the societal attention resulting in a multi-dimensional approach to HCS, provided the basis for an alternative consideration of canine training. An approach based on the notion that the inter-relationship between humans and canines can easily take the form of dogs assisting or working to help humans in various activities. As a result, training changed its approach towards preparing canines to perform many tasks for humans, the most common of which are: herding, pulling loads, protecting, guiding, guarding and assisting police and military. It was discovered that such an approach is feasible and extremely easy to apply. As with the first period, this form of training was applied and earlier when in the first years of the 20th century training dogs for police work was initiated in Germany, and a few years later, during the First World War, the German army relied heavily on the use of German shepherds as ambulance and messenger dogs. But such an organized and accepted canine training has been established after the second World War with the use of guide dogs for the veterans in the United States and continues up to now, providing the impetus of the present training paradigm.

\section{The New Approach}

In the third period, which spans the last few decades, society by accepting that the world must be considered as a dialectic entity, it has realized that today's approach to HCS is not acceptable anymore. In order to express the holistic inter-relationships that operate within HCS, a different approach is required, where HCS is considered as a "whole", which due to its nature operates in the natural world that follows rules expressed by science. As a result, an inter-relationship, as opposed to previous multi-relationship, approach is required that must be scientifically based, in dialectic harmony and respecting all aspects of HCS, integral part of which is training canines and other stakeholders (i.e. owners). Thus, canines within the HCS have an accommodating and clearly more synthetic role. During the last few years, the societal acceptance of the need to consider the world in a dialectic manner and the inter-relationship approach to HCD, have led to a facilitating approach in training. That is, training is focused on canines accommodating human activities or facilitating humans in accomplishing their goals. In this way, the practice of training is a sophisticated process, which molds canines into playing a determining role.

\section{Practicing HCS and Canine Training}

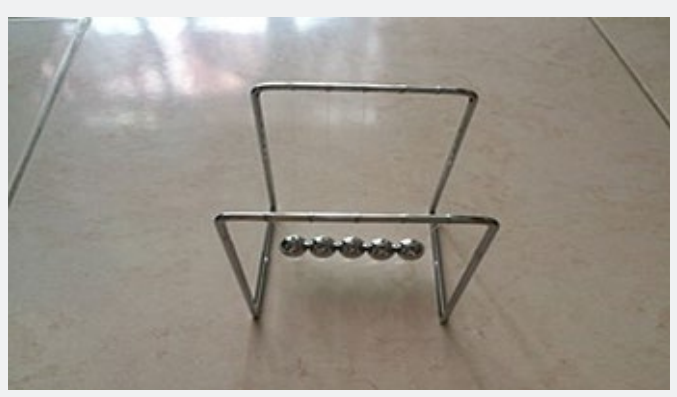

Figure 2: Newton's cradle. 
The way tools and approaches are utilized in canine training depend on the attitudes and the way society regards HCS. Changes in societal values and goals lead into changes in the way newly developed training concepts are practiced and reinforced. That is, every time a societal change is taking place and a new methodological adjustment is imposed on HCS, a new approach must be formulated in order to properly train canines. This implies that over time a distinct process of changes has taken place in the way canine trainings have alter their application mode in response to societal shifts and HCS adjustments. In terms of the way HCS and canine training have been applied, the following three approaches were the most profound (Figure 2, applying synergy and training columns).

\section{The Traditional Practicing Approach}

In the first period, the use of tools utilized within the HCS depended on the social attitudes towards canines. In the traditional practicing approach, the basic societal tenant of HCS as socially non-important activity resulted in a very simple HCS mode. Namely the human-canine interaction [1], which describes a process whereby canines provide empathy in various institutions or at home. It usually takes two basic forms: Animal-Assisted Therapy (to achieve a specific goal) [15], and Animal-Assisted Activities (to provide a more open-ended, spontaneous interaction) [16]. During the same period, the minimal societal attention to canines and the restriction of the HCS application to the simple interaction mode, resulted in a situation where a canine is trained mainly to provide psychological support to people. As a result, canine training was a simple process that did not required any demands and conditions and it was by its nature unstructured with no concerns for planning or design and which characterized the traditional practicing approach.

\section{The Present Practicing Approach}

In the 70's, the second period, the canine community had strongly questioned the mono-dimensional focus of HCS practice on empathy, resulting in the development of alternative ways in practicing the human-canine affiliation. Following changes in societal goals and the way HCS had to be adjusted to them, there was an intense push towards creating a multi-dimensional multi-relationship between humans and canines. An HCS practice approach which is focused on assisting or working for humans by performing various tasks or assisting humans in various activities. It took the form of the human-canine relationship [1], which describes the process whereby canines help humans either as Assistance/ Service dog (performing specific tasks in aiding or assisting humans) or as Working dog (acting as assistants in many human activities). Under this perspective, HCS consisted of the sum of the distinct combinations of canine help expressing the multi-dimensional reality of the present practicing approach. In terms of training, in the second period, the societal changes and the establishment of the relationship form of HCS, has led to a new form of dealing with canines. This new approach was since training necessitates "knowledge" of all the factors and their various aspects that constitute HCS, in order to provide a planned training process. That is, training must be based on a set of procedures and well-designed steps thought in advance and meticulously applied.

\section{The New Practicing Approach}

In the last few years, the third period, due to society's changes and to HCS's adjustments there has been a major alteration of HCS applications, which has been directed towards achieving alternative approaches in applying human-canine affiliations. More specifically, practicing HCS is focused on facilitating human activities or inspiring humans by becoming a tool in achieving scientifically based affiliation goals. As a result, the human-canine inter-relationship has become a sophisticated process, taking the form of the human-canine bonding $[1,17]$, which describes the process whereby canines are facilitating or inspiring human activities, operating within a scientific framework. Under this perspective, the new practicing approaches express the scientific reality of the present-day HCS.

In terms of training, during the last few years the societal acceptance of the human-canine bonding concept has led into: first, formulating a new process by which canines are trained to accommodate humans in fulfilling personal or social needs; and second, that training has to rely on scientific principles to incorporate the accumulated human knowledge that presently determine all human activities. As a result, scientific reasoning is becoming a tool in achieving alternative/nontraditional approaches in training canines to accomplish human goals. In this way, training is a sophisticated process, which molds canines into playing a determining role in the HCS.

\section{Paradigm Shifts}

These changes and their resultant implications are of paramount importance in understanding the formulation of the paradigm shifts in canine training that have taken place during the last few centuries. They basically are addressing the way behavioral modification is achieved within the HCS framework during the three periods mentioned previously (Figure 2, paradigm column). More specifically, in the first period, training was focused on simple specific behavioral modifications (obeying commands) without concern for methods and techniques, which nevertheless were evolving [8]. In addition, the training objective was remote from the daily concerns and interests of the common people, but instead it had to follow mainly the needs and desires of the gentry. The result has been the formulation of the traditional or simple paradigm, whose main training objective has been hunting (Figure 1, first row).

During the second period, training was directed towards changing canine behavior in order to perform useful tasks. This task-oriented training involves applying acquired through training knowledge with the intention to perform real-life tasks useful to humans (from retrieval of dropped items or medication re- 
minders to leading a blind person or discovering narcotics) and which represents a stepwise improvement over the traditional paradigm. The result has been the formulation of the present or behavior-based paradigm, which utilizes a multi-dimensional approach to change composite, advanced and intricate canine behaviors by focusing on both canine behavior modification and human needs (Figure 1, second row).

In the final period, canine training is also focused towards modifying canine behavior, but as a participating factor in supporting human activities. Moreover, training is based on scientific knowledge reflecting the laws and principles that express the world that both humans and canines inhabit. As a result, the affiliation between humans and canines is focused on facilitating human activities or inspiring humans by becoming a tool in achieving alternative, nontraditional scientific approaches. In this way, the practice of the human-canine affiliation is a sophisticated process that leads towards the new or scientific paradigm, which in opposition to the previous paradigms provides for the execution of training based on scientific reasoning. It represents a holistic approach in dialectic harmony with all aspects of HCS that nowadays canine training has to be concerned with. (Figure 1, third row).

In sum, a new canine training paradigm has emerged, which is based on the two pillars of how HCS and training are considered and are practiced. It can be defined as the process of facing HCS or answering human-canine affiliation questions by taking into consideration present-day issues, problems and concerns, which, however, cannot be dealt with adequately by the traditional and the presently used training paradigms. This new scientific training paradigm draws on various perspectives that express the scientific laws and principals that can adequately articulate presentday societal needs and HCS adjustments.

\section{Application: Canine Aggression Training}

Literature and experience have provided a great amount of material presenting, explaining and supporting the two previous canine training paradigms (traditional and present). On the other hand, the proposed new paradigm (scientific) requires some additional exposition in the form of training applications. This is especially necessary in cases with increased difficulty, where training requires approaches not only efficient and effective but mainly fully justified and documented. Such a case is the training for canine aggression.

\section{Canine Aggression}

Canine aggression is defined as the threat of harm to a human, another animal or an inanimate object and involves snarling, growling, snapping, biting, barking or lunging. Therefore, the term "aggression" refers to a wide variety of behaviors that occur for a multitude of reasons in various circumstances. Aggression is not something unusual because virtually all wild animals are aggressive when guarding their territories, defending their offspring and protecting themselves; while species that live in groups, including people and canines, also use aggression and the threat of aggression to keep the peace and to negotiate social interactions.

Independent of the source and the signs of a canine's aggression, the most important thing in training is to understand the nature and the factors that provoke such an action. Experience and the bibliography show: first, that canine aggression is a behavioral problem that can be corrected [18]; and second, that there are many reasons why a canine might be aggressive, which is the result of two main categories: inborne (due to internal factors) and acquired (due to external factors). Aggression due to inborn factors exist mainly because canines exhibit the traits of territoriality and dominance, while the acquired factors result mostly from luck of food and the existence of fear. As a result, knowing and understanding the mechanisms or what the underlying behavioral cause is, makes treatment easier, efficient and more effective [19].

\section{The Scientific Base for Aggression Training: Newton's Laws.}

Most phenomena and conditions in everyday life can be understood or resolved by classical mechanics, which are based on Isaak Newton's contributions. More specifically, Newton's law of motion represents the backbone of mechanics and is expressed by his three physical laws that describe the relationship between a body and the forces acting upon it and it motion in response to those forces. These three laws have been expressed in several ways, over nearly three centuries [20] and can be easily accessed in any mechanics textbook [21].

Newton to demonstrate his laws of motion has developed the Newton's cradle, also known as Newton's Balls or Executive Ball Clicker [22,23]. It is a device that demonstrates inertia and forces as well as conservation of momentum and energy using a series of swinging spheres. A typical Newton's cradle consists of a series of identically sized metal balls suspended in a metal frame so that they are just touching each other at rest. Each ball is attached to the frame by two wires of equal length angled away from each other. This restricts the pendulums' movements to the same plane (Figure 2). When one sphere at the end is lifted and released, it strikes the stationary spheres, transmitting a force through the stationary spheres that pushes the last sphere upward. The last sphere swings back and strikes the still nearly stationary spheres, repeating the effect in the opposite direction.

Theoretically, the Newton's cradle represents an environment where only energy, momentum and gravity are acting on the balls and all the collisions are perfectly elastic, thus the balls would continue to swing forever. However, in real life the movement of the cradle eventually will stop, because a perpetual movement presuppose that the energy of the system is constant, and its transition is not impeded. That is, two sets of factors (internal, associated with the nature of the system and external associated with the operating environment) do not allow these conditions to be met. 


\section{More specifically:}

The major internal factors, which concern exclusively the balls, are related to the necessity of:

i. Perfect elasticity.

ii. Same weight, mass and density of the balls.

iii. Same size.

Some basic external factors, which are concerned with the energy maintenance and its non-impeding transition, are related to the following operations:

i. The swing should be uniform, constant and perfectly aligned.

ii. The movement of the balls and the strings that hold them should be frictionless.

iii. The initial energy should not convert into any other energy form (i.e. to sound energy from the collision of the balls).

iv. The balls should be perfectly aligned, and their position should allow the transfer of energy from one ball to the other with no energy losses.

v. The momentum movement should not be impeded by what in physics is called pressure waves.

In sum, the three main principles of physics related to the conservation of energy, the conservation of momentum and the luck of friction need to operate flawlessly, which is not possible. In the reality of the real-world environment the energy of the system cannot remain constant and its transition cannot be free due to the inability of the above internal and external factors to operate in the ideal way.

\section{Dog Aggression and Newton's Cradle Analogy}

In applying knowledge from science to help canine training, it is suggested that canine aggression can be considered as expressing the energy of the system "canine", which following Newton's cradle cannot remain constant or freely transferred due to internal (associated with the canine) and external (associated with its training) factors. That is, in the same way that the movement of the Newton cradle diminishes, a canine's aggression will diminish when its aggression (the canine system's energy and its transfer) is impeded.

Following this analogy, the following correspondence can be created:

The major internal factors that play a role in the system's energy preservation and transferability and need to be considered are:

i. Canine's fighting spirit (corresponding to perfect ball elasticity).

ii. Canine's weight and mass (in relation to the source of aggression). iii. Canine's size (in relation to the source of aggression).

Major training factors (external) that play a similar role and must be incorporated into the training process are:

i. The canine should face its target on a straight line (corresponding to swing movement).

ii. The source of aggression should be constantly present (corresponding to frictionless movement).

iii. The aggression towards its source should remain constant and undisturbed (corresponding to unconverted energy).

iv. The aggression should not be transformed into other forms of action (corresponding to no loss of energy).

v. The aggression level should remain constant (corresponding to uninterrupted pressure waves).

In other words, training canines should focus on specific actions which are based on the knowledge of scientific facts in order to be efficient and effective and thus provide successful results. More specifically in canine training the following should be utilized:

Internal factors that should be taken into consideration in any effort to improve canine's behavior should include those that the level of risk in living with it and the likelihood of changing its behavior can be revealed through their examination. Such factors are:

i. Canine's fighting spirit which is related to its ability to act as a guide or an aid that can be trusted for protection, alertness, bravery, devotion and constancy in a form that there is more bark than bite. Therefore, this should be considered in training the aggression of a canine.

ii. A canine's weight, mass and density are expressed by its age. Indeed, young dogs with an aggression problem are believed to be more malleable and easier to treat than older dogs and therefore should be taken into consideration.

iii. Regardless of other factors, large dogs are more frightening and can inflict more damage than small dogs and should be taken into consideration.

In training a canine in order to modify its aggressive behavior, which can be considered the external factors in this process, the following training actions should be considered for a successful canine aggression modification:

i. The canine must be removed from its initial position and forced to face the source of his aggression not in a straight line. In this way the transfer of energy of the canine system or the canine aggression will be impeded.

ii. The source aggression should be removed during training. This creates a form of friction that constrains the energy of the system or decreases the aggression of the canine. 
iii. The attention of the canine should be diverted towards a controlled object (i.e. a ball). Such an action during training results in the transfer of the canine system's energy to a different form and lead to its decrease.

iv. The pray should be transformed, which is achieved by the removal of the canine's food. In this way the food removal creates a friction that impedes the transfer of energy and thus diminishes the canine's aggression.

v. A host of actions should be taken leading towards a decrease in the level (impulse/pressure) of aggression.

\section{Conclusion}

Animals have been used for centuries to enhance human interest and written information about training canines to facilitate that goal dates back at least to Roman times [24]. But canine training in the present form of purposeful changing a dog's behavior to become household pet has been developed after the second WW in response to the needs of war veterans, the increasing complexities of suburban living and the increased interest in developing humane canine training methods [25]. The result has been the ever increasing of what Koehler has termed the "prattle of dog psychologists" [25], which ignores that fragmented non-holistic approaches to canine psychology, without regard to the socioeconomic, political, cultural etc. conditions, cannot produce successful training methods. Indeed, it is no longer possible to look for precursors of modern ideas in canine training without stressing the changing social attitudes toward them.

The previous discussion validates a universal thesis that is also appropriate in canine training, namely: that the best advice is to be sensitive to areas from which change is emerging. Indeed, in the past decades changes in societal values towards HCS and canine training (from considering them as having limited individual and societal value, to reaching a practical recognition by the society and to considering them as a dialectic entity) have led to changes: first, on how we consider HCS (from passive, to acting and to determining) and training (from hunting, to helping, and to accommodating); and second on how we practice HCS (from integration, to relationship and to bonding) and training (from unstructured, to planned and to scientific).

The new canine training paradigm is basically characterized by its scientific nature, which makes it a genuine revolutionary step in canine training. But most importantly it formulates: first, a connection between determining HCS in scientific terms with accommodating training by considering real life conditions; and second by scientifically applying the concept of bonding in HCS with integrating societal concerns in training. As a result, the new paradigm should be understood as representing the confrontation between training and the scientific principles of the real world. But out of this phenomenological confrontation rises a situation which is too broad to be handled by passive or simple active approaches or by interaction or relationship methods, with no regard for the holistic nature of the world. That is, the purpose of the scientific paradigm is more than just to address questions that transect behavior modification approaches or technics and methods in achieving canine training. It involves a spectrum of scientific principles to help the HCS system to determine when and how to confront canine training within the existing societal value system.

In sum, in the last few decades there have been two pedagogic shifts from the traditional or simple paradigm, to presently used behavior-based paradigm, bringing canine training to the scientific paradigm. But most importantly, which is the thesis and contribution of this paper, is that the new paradigm in canine training is necessary to overcome lack of the necessary integration in the scientifically shallow present paradigm, in order to meet present day scientific, societal and training environment. Finally, in order to illustrate the new paradigm, an example is presented explaining the way canines can be trained to restrain their aggression by utilizing scientific knowledge. More specifically, considering that canine aggression corresponds with the scientific principles of the Newton's cradle requiring that the energy (aggression) of the canine can be diminish if its training includes: first, the consideration of a set of internal factors that impact on a canine's behavior; and second, by applying specific training actions that scientifically guarantee a successful canine aggression modification. In other words, training based on scientific knowledge reflecting the laws and principles that express the world that both humans and canines inhabit can result in an effective, efficient and mainly scientifically justified training approach.

\section{Conflicts of Interest}

The authors declare no conflict of interest.

\section{References}

1. Koutsopoulos T, Koutsopoulos CK (2018) A Holistic Approach to Human-Canine Synergy in Education. Psychol Behav Sci Int J 9(2): 1-5.

2. Birbil C, Theo Koutsopoulos, Cathy Makropoulos, Alessandra Sax, Stefanos Gialamas, et. al. (2015) Dogs in learning: Transforming Education. Inter J Elem Educ 4(2): 16-24.

3. Koutsopoulos CK, Gialamas S, Koutsopoulos T (2016) A true manifestation of Mp: Dogs in Learning. In: M Avgerinou, S Gialamas (Eds). Revolutionizing K-12 Blended Learning through the i2 Flex Classroom Model. IGI Global, Hershey, PA 27- 38.

4. Furtado C (1963) The Economic Growth of Brazil: A Survey from Colonial to Modern Times. University of California Press, Los Angeles, USA.

5. Burch MR (2012) The Evolution of Modern-Day Dog Training. National Animal Interest Alliance.

6. Koutsopoulos CK, Kotsanis Y (2014) School on Cloud: Towards a paradigm shift. Themes in Science and Technology Education. 7(1): 47-62.

7. Kuhn TS (1970) The Structure of Scientific Revolutions (2 $2^{\text {nd }}$ Edn). University of Chicago Press, Chicago, USA.

8. Bob BM Wong (2015) Behavioral responses to changing environments. Behavioral Ecology, Candolin, U 26(3): 665-673. 
9. Burch MR, Bailey J S (1999) How Dogs Learn. Howell Book House. New York, USA.

10. Hutchinson WN (1965) Dog Breaking for the Gun: The Most Expeditious, Certain and Easy Method, With Copious Notes on Shooting Sports. Vintage Dog Books, New York, USA.

11. Millan C, Peltier M (2010) Cesar's Rules. Three Rivers Press, New York, USA.

12. Bustad KL (1980) Animals, aging and the Aged. University of Minnesota Press, Minneapolis, USA.

13. Nightingale F (1860) Notes on Nursing: What it is and what it is not. D. Appleton and Company, New York (NY), USA,

14. Koutsopoulos T, Koutsopoulos KC (2018) Dogs in Learning: An Implementation of Human-Canine Synergy in Education. J Edu Soc Behav Sci 25(2): 1-12.

15. Brodie JS, Biley CF (1999) An exploration of the potential benefits of pet-facilitated therapy. Journal of Clinical Nursing 8(4): 329-337.

16. Fine HA (2008) Handbook on animal-assisted therapy: theoretical foundations and guidelines. Academic Press, New York, USA,
17. American Society for the Prevention of Cruelty to Animals Aggression(2019).

18. Koutsopoulos T (2019) Today's Society: Exploring Dogs. Disigma Publishing, Thessaloniki, Greece.

19. Newton I (1829) The mathematical principles of Natural Philosophy. Benjamin, London, UK.

20. Kleppner D, Kolenkow R (2019) Introduction to Mechanics (3 ${ }^{\text {rd }}$ Edn). Cambridge University Press, Cambridge, UK.

21. Harvard Natural Sciences Lecture Demonstrations (2016) Newton's Cradle. Harvard University, Harvard, USA.

22. Palermo, E (2019) How Does Newton's Cradle Work? Live Science.

23. Goodlad J (1984) A place called school. McGraw-Hill Book Company, New York, USA.

24. Bihm EM, Gillaspy JA (2012) Marian Breland Bailey (1920-2001). The Encyclopedia of Arkansas History and Culture. The Central Arkansas Library System. Little rock, USA.

25. Koehler W (1962) Koehler Method of Dog Training. Howell books: New York, USA.

\section{Your next submission with Juniper Publishers will reach you the below assets}

- Quality Editorial service

- Swift Peer Review

- Reprints availability

- E-prints Service

- Manuscript Podcast for convenient understanding

- Global attainment for your research

- Manuscript accessibility in different formats

( Pdf, E-pub, Full Text, Audio)

- Unceasing customer service

Track the below URL for one-step submission https://juniperpublishers.com/online-submission.php 